\title{
Influencia de la proporción sexual y del ambiente en la tasa de crecimiento poblacional de Callinectes bellicosus (Decapoda: Portunidae) del Golfo de California
}

\author{
Demetrio Rodríguez-Félix ${ }^{1}$, Miguel Ángel Cisneros-Mata, ${ }^{2,}$, Eugenio Alberto Aragón-Noriega ${ }^{1}$ \\ \& José Alfredo Arreola-Lizárraga ${ }^{1}$ \\ 1. Centro de Investigaciones Biológicas del Noroeste, Unidad Sonora. Km 2.35 Camino al Tular, Estero Bacochibampo, \\ Guaymas, Sonora 85454, México; demetrior@cibnor.mx, aaragon04@cibnor.mx, aarreola04@cibnor.mx \\ 2. Instituto Nacional de Pesca. CRIP Guaymas. Calle 20 No. 605-Sur. Guaymas, Sonora 85400, México; \\ miguel.cisneros@inapesca.gob.mx \\ * Correspondencia
}

Recibido 27-VII-2015. Corregido 10-III-2016. Aceptado 01-IV-2016.

\begin{abstract}
Sex ratio and environmental influence on population growth rate of Callinectes bellicosus (Decapoda: Portunidae) in the Gulf of California. The brown swimming crab (Callinectes bellicosus) is an economically important species in the Gulf of California, and its fishing activity, held in Sonora from 1986, has been affected by a 20 -year declining trend in its biomass. With the aim to understand the possible reasons of this species population changes along time, we estimated population growth rate (r) and sex ratio of C. bellicosus, and combined them with three parameters describing its habitat: sea temperature, wetland extension and habitat size in four areas along the coast of Sonora. For this, monthly mean sex ratio was estimated from crabs samples obtained from commercial catches during 1998-2002 and 2012; mean sea surface temperature for the spawning period (May-August) were derived from remote sensors for the same years; while wetland coverages were obtained from published reports, and habitat size was estimated as the fishing surface. For each area, $r$ was estimated using a method developed for limited data situations using commercial landings ( $t$ ) from 1986-2013. With data from the four areas, simple and multiple linear regression models were developed to ascertain theoretical sensitivities of $r$ to variations in sex ratio and environmental parameters. A total of 24556 crabs were sampled; males dominated $(68.8 \%)$ over females during the study period and in all areas; a cluster analysis identified two groups according to sex ratio: a Northern group with zones 1 and 2, and a Southern group with zones 3 and 4. r values were different in all zones $(\mathrm{P}<0.001)$ as was sex ratio $(\mathrm{P}=0.037)$; no differences in temperature were identified within the study years $(\mathrm{P}>0.995)$. Both the estimated data and sensitivity analyses suggest the existence of a direct and positive dependence of $r$ on the proportion of female crabs and wetland size. We hypothesize that excess fishing of females caused the declining biomass trend of the brown swimming crab in Sonora, and concluded on the convenience of implementing harvest refugia inside coastal wetlands to protect females during the spawning season. Rev. Biol. Trop. 64 (3): 1259-1271. Epub 2016 September 01.
\end{abstract}

Key words: Callinectes bellicosus, sex ratio, population growth rate, Gulf of California.

La jaiba café, Callinectes bellicosus (Stimpson, 1859) habita en fondos blandos costeros, desde el sur de California, E.U.A. hasta el golfo de Tehuantepec, incluido el Golfo de California, México (Hendrickx, 1984). Sus poblaciones parecen tener distribución heterogénea por tamaños y sexos en aguas con temperatura de 20 a $32{ }^{\circ} \mathrm{C}$ (Arreola et al., 2003; Castro \& DeAlteris, 1988; Estrada, 1999). Los adultos de $C$. bellicosus son eurihalinos y sus larvas requieren salinidades del ambiente marino; por ello las hembras grávidas liberan los huevecillos en las bocas de lagunas costeras y esteros (Fischer \& Wolff, 2006; Sánchez \& Gómez, 1992).

Se conoce poco sobre la influencia del ambiente en las poblaciones de jaibas. La abundancia de hembras ovígeras se asocia a 
humedales (Carmona, 2009), pero no existen estudios sobre la relación entre el tamaño de estos, la proporción de sexos y la tasa de crecimiento poblacional (r). La proporción de sexos es importante porque la población requiere una cantidad de hembras que genere la producción de huevos adecuada (Lasker, 1985) y suficientes machos para evitar limitación espermática (Carver, Wolcott, Wolcott, \& Hines, 2005). Por lo tanto, la proporción de sexos debe optimizar la adecuación ("fitness") de la jaiba a su medio ambiente. Una medida de adecuación es $r$, que se relaciona con el ambiente (Stearns, 1992).

En Sonora, México, C. bellicosus sostiene una pesquería artesanal con producción media de 4500 toneladas en los últimos cinco años. Los juveniles son capturados en lagunas costeras; adultos, incluyendo hembras ovígeras son capturados en las lagunas y en el litoral marino. Análisis recientes evidenciaron que desde hace dos décadas la biomasa va en descenso (Cisneros et al., 2014). Para contrarrestar los efectos de la pesca, se decretó talla mínima de $11.5 \mathrm{~cm}$ de ancho de caparazón y se prohibió capturar hembras grávidas (DOF, 2006). A partir de 2013 se implementó veda total del 1 mayo al 30 junio y veda de hembras del 1 julio al 31 agosto (DOF, 2012).

Aunque la proporción media de hembras y machos esté en equilibrio (1:1) varias especies de crustáceos presentan segregación por sexos (Baptista, Pinheiro, Blankensteyn, \& Borzone, 2005; Branco \& Masunari, 2000). Por ello el manejo pesquero debe considerar la estructura de las poblaciones y evaluar el efecto de la pesca selectiva sobre tallas o sexos (Grabowski, Morilhas, \& Leão, 2014; Orensanz, Armstrong, Armstrong, \& Hilborn, 1998; Sato, 2012). El objetivo general de este estudio fue determinar variaciones en la proporción sexual, la tasa de crecimiento poblacional, y evaluar la relación de la tasa de crecimiento de $C$. bellicosus con su hábitat en el litoral de Sonora, México, con el fin último de explorar tácticas de manejo pesquero.

\section{MATERIALES Y MÉTODOS}

Área de estudio: Con extensión de 1200 $\mathrm{km}$, la costa de Sonora conforma la parte norte del margen oriental del Golfo de California. Sonora tiene diez humedales costeros con 71 529 ha; en el norte reciben poca agua dulce, a diferencia del centro-sur donde una serie continua de lagunas reciben agua de precipitación pluvial, drenajes agrícolas, y los ríos Yaqui y Mayo (Arreola, 1995).

Para este trabajo, el área de estudio se dividió en cuatro zonas, considerando la variabilidad del litoral de Sonora, que incluyen los siguientes sitios, ordenados latitudinalmente de norte a sur (Fig. 1): 1) Puerto Peñasco, Campodónico y El Jagüey; 2) Punta Chueca, Bahía de Kino, El Sahuímaro y El Choyudo; 3) Playa del Sol, Bahía Las Guásimas y Bahía de Lobos; y 4) Bahía El Tóbari, Bahía de Yavaros y Bahía de Agiabampo.

Muestreo: La proporción de sexo de jaiba café se obtuvo de muestreos mensuales de descargas comerciales y a bordo de embarcaciones artesanales que efectuaron sus actividades, disponibles para 1998-2002 y 2012. La jaiba se pesca con diversos artes, pero en este estudio se consideró muestreos de capturas con trampas tipo Chesapeake (Cisneros et al., 2014). En promedio se utilizaron 80 trampas por embarcación, caladas mayormente ( $>80 \%$ ) de una a cinco brazas en bahías, y hasta 10 brazas en la ribera marina (DOF, 2014).

Proporción de sexo: Se estimó la proporción mensual hembras/machos $(\mathrm{H} / \mathrm{M})$ por zona. En los meses sin datos, la proporción $\mathrm{H} / \mathrm{M}$ se obtuvo por interpolación de valores contiguos; cuando faltaban dos datos, el primero se estimó con la tasa de cambio promedio de tres meses consecutivos y el segundo por interpolación lineal (Izar, 1998). Con el cuadro completo $\mathrm{H} / \mathrm{M}$ por mes y zona, se realizaron análisis posteriores. 


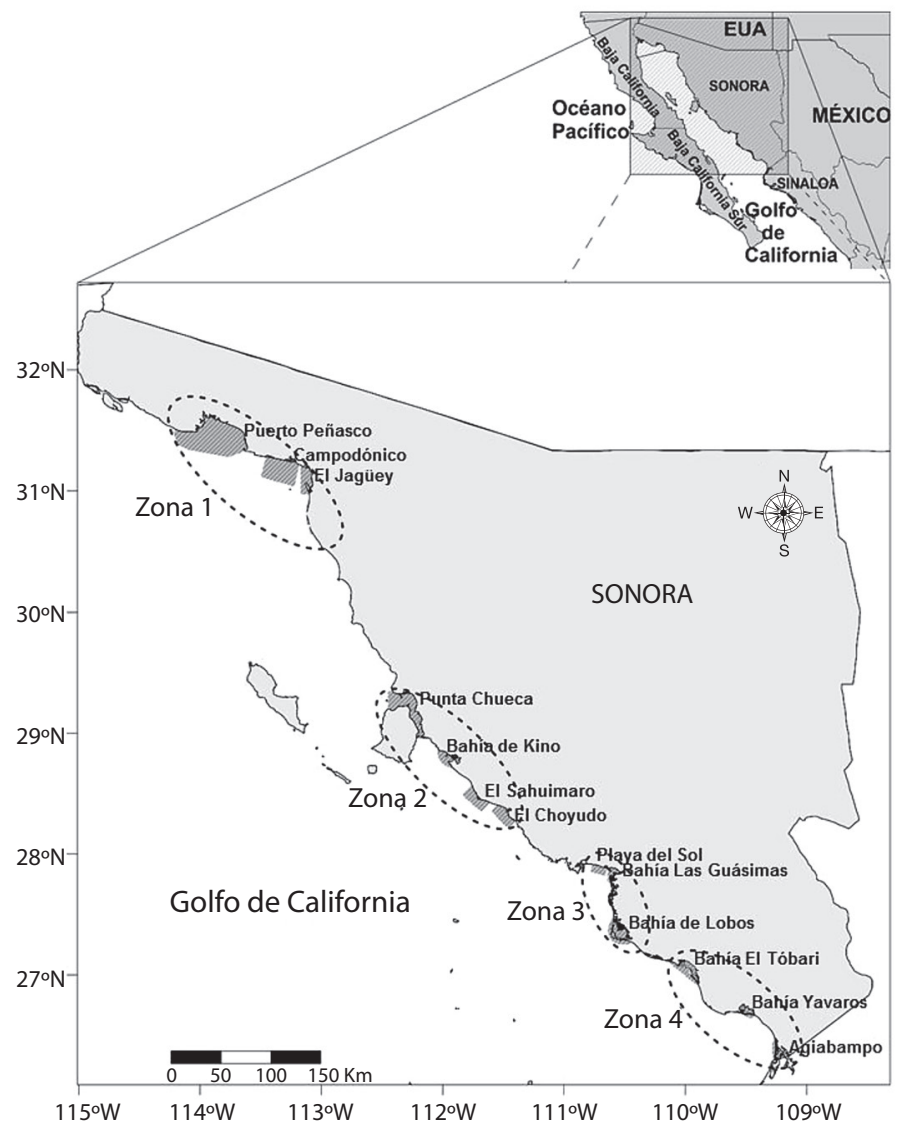

Fig. 1. Área de estudio con las 13 comunidades de pesca de C. bellicosus en cuatro zonas de la costa de Sonora, México. No hay pesca de jaiba entre zonas (DOF, 2014).

Fig. 1. Study area showing 13 C. bellicosus fishing communities in four zones in the coast of Sonora, Mexico. There is no crab fishing between zones (DOF, 2014).

Las diferencias de H/M entre años se probaron por tabla de contingencia con la prueba $\chi^{2}$ y entre zonas con el test de Kruskal-Wallis y comparaciones Mann-Whitney a posteriori (Zar, 1999). Se construyó un dendrograma de similitud de $\mathrm{H} / \mathrm{M}$ por zona utilizado la distancia euclidiana (Elmore \& Richman, 2001). Estos análisis se realizaron en el programa PAST (Hammer, Harper, \& Ryan, 2001).

Crecimiento poblacional: Se estimaron las $\mathrm{r}$ por zona mediante el método de Martell y Froese (2012) utilizando datos oficiales de captura comercial $(\mathrm{t})$ de 1986-2013 y estimaciones de la resiliencia de las poblaciones de jaiba, consideradas como cuatro stocks. Se asumió que en 1986 la biomasa de los cuatro stocks estaban entre 75-100\% de sus capacidades de soporte (K), y en 2013 entre 60-85\% de K (Martell \& Froese, 2012). Lo anterior, considerando que la pesca comenzó en la década de 1980 (Nevárez et al., 2003) y en tres décadas se estabilizó alrededor de 4500 t (Cisneros et al., 2014). En todos los casos $r$ varió entre 0.2/año y 1.8/año.

Se incluyó error de proceso de $5 \%$ y de observación con $\mathrm{CV}=5 \%$ en la captura comercial (Hilborn \& Walters, 1992). El error de proceso se refiere a la naturaleza estocástica de la estimación de la biomasa; el de observación 
reconoce la variabilidad en los registros de captura $\left(\mathrm{C}_{\mathrm{t}}^{\text {obs }}\right.$ ); la captura se muestreó de una distribución Normal con media $\mathrm{C}_{\mathrm{t}}^{\mathrm{obs}} \mathrm{y}$ varianza $=0.025$. En 20000 simulaciones Monte Carlo se generaron valores aleatorios de $\mathrm{r}$ y en los modelos de regresión sus medianas se consideraron como valores observados. Mediante el test de Kruskal-Wallis se compararon 4000 valores de $r$ por zona elegidos al azar para probar la igualdad de sus medianas (Zar, 1999).

Relación entre $\mathbf{r}, \mathrm{H} / \mathrm{M}$ e indicadores ambientales: Los modelos de regresión lineal simple (MRLS) y múltiple (MRLM) para $\mathrm{r}$ en función de $\mathrm{H} / \mathrm{M}$ consideraron las siguientes variables ambientales:

TSM: Promedio mensual de mayo a agosto de la temperatura superficial del mar en la zona costera, de sensores remotos. En esos meses ocurre el desove de la jaiba café (DOF, 2012). La TSM para 1998-2002 y 2012 se obtuvo del Comprehensive Oceanic and Atmospheric Data Set del NOAA-CIRES Climate Diagnostics Center. La interpolación se genera semanalmente para cuadrantes de un grado de latitudlongitud (Reynolds, Rayner, Smith, Stokes, \& Wang, 2002). Al ser la zona de pesca de jaiba muy costera, por la fuerte mezcla de marea y vientos (Marinone \& Lavin, 1997) se asumió que la TSM es indicativa del ambiente de la jaiba café.

SP: Tamaño del área de distribución de la jaiba, considerada como la superficie cubierta por las flotas de pesca (estimado en el presente trabajo). Esto supone que $\mathrm{K}$ depende del área de distribución de la jaiba y que se relaciona con $r$ (Schaefer, 1954).

SH: Superficie de humedales (Ayala, Malpica, \& Gutiérrez, 1980; Ayala, Gutiérrez, \& Malpica, 1990; Beman \& Christopher, 2006; Canchola, 2009; Cruz \& Ortega, 1991; Guzmán, 1993;Ortega \& Vázquez, 1989).

Se desarrollaron cuatro MRLS para $r$ en función de: a) H/M, b) TSM, c) AH, y d) SP. Ocho MRLM fueron para $r$ en función de: e) $\mathrm{H} / \mathrm{M}$ y SP, f) H/M y TSM, g) H/M y SH, h) $\mathrm{H} / \mathrm{M}$ y un índice compuesto $\mathrm{TSM} / \mathrm{SP} / \mathrm{SH}$, i) $\mathrm{H} / \mathrm{M}$ y un índice compuesto TSM/SH, j) SP y
$\mathrm{SH}, \mathrm{k}) \mathrm{SP}$ y un índice compuesto (H/M)/TSM, y l) un índice compuesto SP/SH y otro (H/M)/ TSM. Los parámetros se obtuvieron por máxima verosimilitud (Hilborn \& Mangel, 1997). La log-verosimilitud (LL) se estimó como sigue (Punt \& Hilborn, 2001):

$$
\mathrm{LL}=\mathrm{e}^{\frac{-\sum_{1}^{4}\left[\ln \left(\mathrm{r}_{\text {obs }}\right)-\ln \left(\mathrm{r}_{\text {est }}\right)\right]^{2}}{2 \sigma^{2}}}
$$

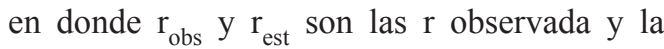
estimada con los modelos; $\sigma$ es la desviación estándar de los errores de $\mathrm{r}_{\text {obs }}$.

Los MRLS se eligieron con el Criterio de Información de Akaike (AIC), y los MRLM con el AIC corregido (AICc) para muestras pequeñas $(\mathrm{n} / \mathrm{k}<40)$ (Burnham \& Anderson, 2002):

$$
\operatorname{AICc}=\operatorname{AIC}+\frac{2 \mathrm{k}(\mathrm{k}+1)}{\mathrm{n}-\mathrm{k}-1}
$$

en donde $\mathrm{AIC}=2 \mathrm{k}-2 \ln (\mathrm{LL}), \mathrm{k}=$ número de parámetros incluyendo $\sigma, \mathrm{y} \mathrm{n}=$ cantidad de datos. En los MRLS no se utilizó AICc porque $\mathrm{n}-\mathrm{k}-1=0$. En los MRLM, $\mathrm{k}=4$ (una constante y dos coeficientes de los modelos, además de $\sigma)$ y $\mathrm{n}=4$ (cuatro zonas). Para los MRLM, con los valores de AICc se determinó la diferencia entre cada uno de los valores de AICc y el valor mínimo: $\Delta_{\mathrm{i}}=\mathrm{AICc}_{\mathrm{i}}-\mathrm{AICc}_{\text {mín }}$, y la bondad relativa de los modelos con los pesos normalizados de Akaike (Burnham \& Anderson, 2002):

$$
\mathrm{w}_{\mathrm{i}}=\frac{\mathrm{e}^{-0.5 \Delta_{\mathrm{i}}}}{\sum_{\mathrm{i}=1}^{5} \mathrm{e}^{-0.5 \Delta_{\mathrm{i}}}}
$$

Con los modelos de menor AIC o AICc se evaluó la sensibilidad teórica de $\mathrm{r}$ a cambios en $\mathrm{H} / \mathrm{M}$ y en los parámetros ambientales, bajo los siguientes escenarios: 1) $\pm 3^{\circ} \mathrm{C}$ en TSM; 2) \pm $30 \%$ en H/M; y 3$) \pm 30 \%$ en SP.

\section{RESULTADOS}

No se encontraron diferencias significativas en la TSM entre años (Kruskal-Wallis, $0.283<\mathrm{H}<0.606 ; \mathrm{P}>0.995)$. Se analizaron 24556 organismos durante 1998-2002 y 2012 
(Cuadro 1). En el cuadro 2 se muestran las H/M indicando los 14 (29.2\%) datos faltantes estimados por interpolación. La $(\mathrm{H} / \mathrm{M})$ no difiere $\left(\chi^{2}=0.27 ; \mathrm{P}=0.6\right)$ en el período de $1998-2002$ (0.40) comparado con 2012 (0.45). En general, $\mathrm{H} / \mathrm{M}$ fue $29.2 \%(0.54 \mathrm{H}: 1.00 \mathrm{M})$, no es homogénea entre zonas $\left(\chi^{2}=8.5 ; \mathrm{P}=0.037\right)$ y varía mensualmente de manera distinta (Fig. 2). La zona 3 presentó la $\mathrm{H} / \mathrm{M}$ más cercana a $1: 1$ y la r mayor (0.59/año) (Cuadro 3); la zona 4 fue segunda en el valor $\mathrm{H} / \mathrm{M}$ y mostró la menor $\mathrm{r}$ (0.30/año). En las zonas 3 y 4 tanto SH como SP fueron mayores; la TSM aumentó de norte a sur. En general, H/M, TSM y SH son mayores en las dos zonas del sur que en las dos del norte. El análisis de similitud basado en $\mathrm{H} / \mathrm{M}$

\section{CUADRO 1}

Número total mensual de C. bellicosus muestreadas por zona durante 1998-2002 y 2012

TABLE 1

Monthly total number of C. bellicosus sampled at each zone during 1998-2002 and 2012

\begin{tabular}{ccccccccccccccccccccccccccccc} 
Mes & \multicolumn{1}{c}{ E } & \multicolumn{1}{c}{ F } & \multicolumn{1}{c}{ M } & \multicolumn{1}{c}{ A } & M & J & \multicolumn{1}{c}{ J } & \multicolumn{1}{c}{ A } & \multicolumn{1}{c}{ S } & \multicolumn{1}{c}{ O } & N & D \\
Zona & M & H & M & H & M & H & M & H & M & H & M & H & M & H & M & H & M & H & M & H & M & H & M & H \\
$\mathbf{1}$ & 0 & 0 & 0 & 0 & 28 & 106 & 161 & 91 & 0 & 0 & 0 & 0 & 91 & 89 & 168 & 194 & 135 & 75 & 138 & 5 & 378 & 139 & 0 & 0 \\
$\mathbf{2}$ & 1004 & 133 & 692 & 28 & 1178 & 58 & 181 & 27 & 0 & 0 & 0 & 0 & 829 & 362 & 2767 & 617 & 2083 & 906 & 1652 & 646 & 1547 & 287 & 1434 & 814 \\
$\mathbf{3}$ & 28 & 10 & 23 & 22 & 0 & 0 & 22 & 39 & 44 & 23 & 30 & 15 & 267 & 140 & 608 & 384 & 0 & 0 & 254 & 201 & 71 & 46 & 96 & 62 \\
$\mathbf{4}$ & 0 & 0 & 93 & 28 & 0 & 0 & 31 & 34 & 0 & 0 & 0 & 0 & 0 & 0 & 551 & 224 & 144 & 101 & 359 & 218 & 136 & 126 & 26 & 22 \\
\hline
\end{tabular}

CUADRO 2

Proporción mensual hembras/machos de C. bellicosus por zona

TABLE 2

Monthly female/male proportion of $C$. bellicosus by zone

\begin{tabular}{cccccccccccccc} 
ZONA & $\mathrm{E}$ & $\mathrm{F}$ & $\mathrm{M}$ & $\mathrm{A}$ & $\mathrm{M}$ & $\mathrm{J}$ & $\mathrm{J}$ & $\mathrm{A}$ & $\mathrm{S}$ & $\mathrm{O}$ & $\mathrm{N}$ & $\mathrm{D}$ \\
1 & $\underline{0.17}$ & $\underline{0.25}$ & 0.38 & 0.57 & $\underline{0.70}$ & $\underline{0.83}$ & 0.98 & 1.15 & 0.56 & 0.04 & 0.37 & $\underline{0.27}$ \\
2 & 0.13 & 0.41 & 0.49 & 0.15 & $\underline{\underline{0.39}}$ & $\underline{0.62}$ & 0.44 & 0.22 & 0.43 & 0.39 & 0.19 & 0.57 \\
3 & 0.36 & 0.96 & $\underline{1.36}$ & 1.77 & 0.52 & 0.50 & 0.52 & 0.63 & $\underline{0.71}$ & 0.79 & 0.65 & 0.65 \\
4 & $\underline{0.57}$ & 0.30 & $\underline{0.70}$ & 1.10 & $\underline{1.61}$ & $\underline{0.98}$ & $\underline{0.35}$ & 0.41 & 0.70 & 0.61 & 0.93 & 0.85 \\
\hline
\end{tabular}

Las cifras subrayadas y en cursivas indican los valores interpolados.

Numbers in italics and underlined indicate interpolated values.

CUADRO 3

Valores de los parámetros usados para desarrollar los modelos de regresión lineal por zona en el litoral de Sonora

TABLE 3

Parameter values used to develop linear regression models by zone in the coast of Sonora

\begin{tabular}{cccccr} 
Zona & $\mathrm{r}_{\text {obs }}(/ \mathrm{año})$ & $\mathrm{H} / \mathrm{M}$ & $\mathrm{SP}\left(\mathrm{km}^{2}\right)$ & $\mathrm{TSM}\left({ }^{\circ} \mathrm{C}\right)$ & $\mathrm{SH}\left(\mathrm{km}^{2}\right)^{*}$ \\
1 & 0.34 & 0.45 & 147.73 & 27.04 & 15.89 \\
2 & 0.39 & 0.35 & 121.25 & 28.00 & 2.53 \\
3 & 0.59 & 0.72 & 268.98 & 28.46 & 354.56 \\
4 & 0.30 & 0.69 & 269.13 & 29.03 & 342.30 \\
\hline
\end{tabular}

$\mathrm{r}_{\mathrm{obs}}=$ tasa de crecimiento poblacional, $\mathrm{H} / \mathrm{M}=$ proporción hembras/machos, $\mathrm{SP}=$ superficie de pesca, $\mathrm{TSM}=$ temperatura superficial del mar costera, y $\mathrm{SH}=$ superficie de humedales.

* Referencias: Ayala et al., 1980; 1990; Ortega et al., 1988; Ortega \& Vázquez, 1989; Cruz \& Ortega, 1991; Guzmán, 1993; Beman \& Christopher, 2006; Canchola, 2009. 


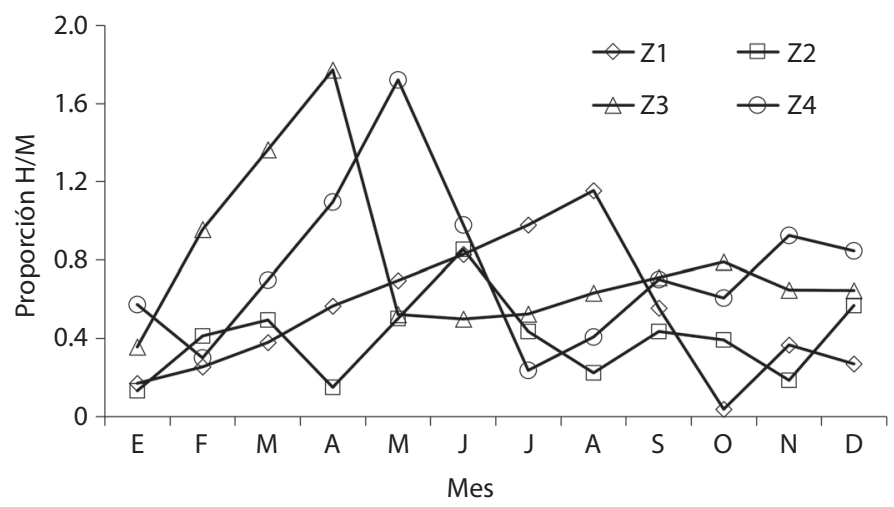

Fig. 2. Proporción mensual de hembras/machos de C. bellicosus en cuatro zonas consideradas en el presente estudio.

Fig. 2. Monthly proportion of female/male of C. bellicosus in four zones considered in the present study.

identificó dos grupos: Las zonas 1 y 2 al norte, y las zonas 3 y 4 al sur de Sonora (Fig. 3). La proporción $\mathrm{H} / \mathrm{M}$ por estación del año fue similar en las zonas 1 y 2, con máximo en verano; las zonas 3 y 4 fueron distintas, con máximo en primavera (Fig. 4).

Las distribuciones de $\mathrm{r}$ para las cuatro zonas (Fig. 5) fueron distintas (Kruskal-Wallis, $\mathrm{H}=2$ 410; $\mathrm{P}<0.001)$; las comparaciones $a$ posteriori (Mann-Whitney) también indicaron que las medianas de $\mathrm{r}$ son distintas para cada

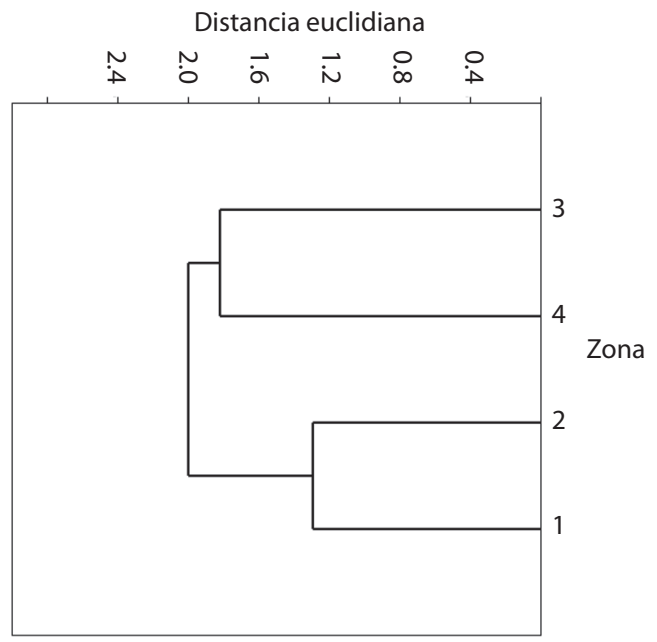

Fig. 3. Agrupación de las zonas de estudio con base en la proporción sexual de C. bellicosus en el litoral de Sonora, México.

Fig. 3. Clustering of the study zones based on the sex ratio of $C$. bellicosus in the coast of Sonora, Mexico. zona $(\mathrm{P}<0.001)$. La mediana de $\mathrm{r}$ de los cuatro stocks osciló entre 0.30 y 0.59 /año, con máximo en la zona 3 y mínimo en la 4 . Los modelos con menor AIC fueron los MRLS (Cuadro 4). Los dos mejores MRLS son el b y el d, que relacionan $\mathrm{r}$ con TSM y SP, respectivamente, con ecuaciones: $\mathrm{r}_{\mathrm{b}}=0.024$ (TSM)-0.27 y $\mathrm{r}_{\mathrm{d}}=$ $0.00055(\mathrm{SP})+0.2948$. El análisis de sensibilidad indicó una relación directa entre TSM y $r$ en las cuatro zonas; en el caso de SP la relación fue directa solo en las zonas 3 y 4 (Fig. 6).

Los pesos relativos de los cuatro MRLM fueron iguales $\left(\mathrm{w}_{\mathrm{i}} \cong 12.5 \%\right)$ y por ello se optó por los modelos j y k, de menor AICc: $r_{j}=$ $1.0692-0.0054228(\mathrm{SP})+0.0024044(\mathrm{SH}), \mathrm{y} \mathrm{r}_{\mathrm{k}}$ $=0.14496-0.00013249(\mathrm{SP})+15.522[(\mathrm{H} / \mathrm{M}) /$ TSM]. Con el primer MRLM se analizó la sensibilidad de $r$ a cambios en $\mathrm{SH}$, y con el

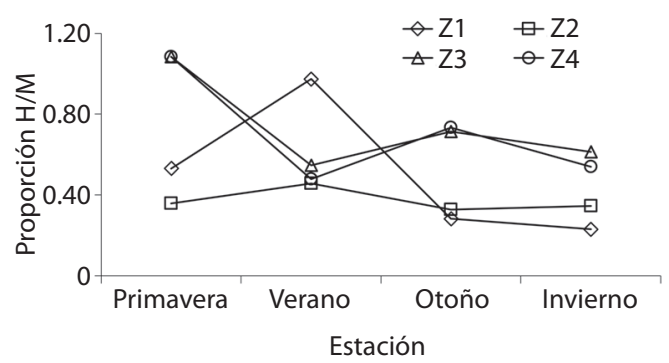

Fig. 4. Variación estacional de la proporción hembras/ machos de C. bellicosus en las cuatro zonas de estudio.

Fig. 4. Seasonal variation of female/male ratio of $C$. bellicosus in the four study zones. 


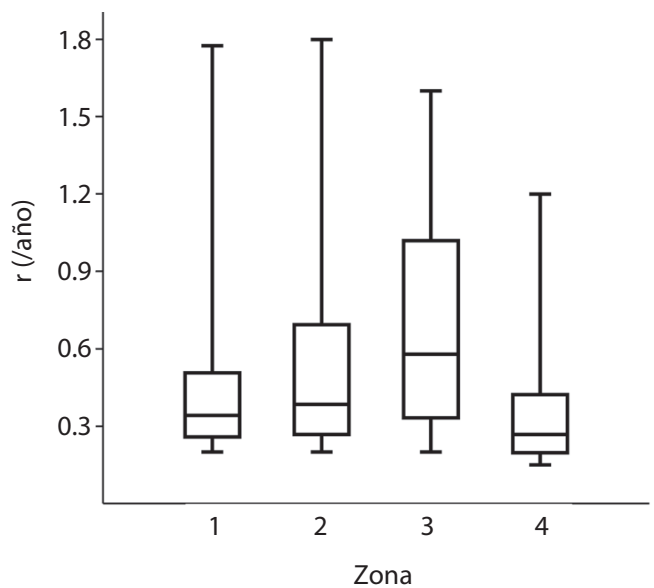

segundo la respuesta a cambios en SP, H/M y TSM. Con los MRLM, en las cuatro zonas $\mathrm{r}$ varió en relación directa con $\mathrm{H} / \mathrm{M}$ (Fig. 7A) e inversa con TSM (Fig. 7B). Por otro lado, $r$ varió en relación directa con $\mathrm{SH}$ en las zonas 3
Fig. 5. Diagramas de caja para 4000 valores de $\mathrm{r}$ estimados por zona para $C$. bellicosus. La línea interna es la mediana (50\% de la frecuencia acumulada), los extremos de los rectángulos los cuartiles 1 (25\%) y $3(75 \%)$ y las barras exteriores los máximos y mínimos.

Fig. 5. Box plots of $4000 \mathrm{r}$ values estimated per zone for C. bellicosus. Inner lines are medians ( $50 \%$ of cumulative frequency), extremes of the rectangles are quantiles 1 $(25 \%)$ and $3(75 \%)$, and outer bars indicate minimum and maximum.

y 4 (Fig. 7D); en ninguna zona la variación de SP generó cambios en r (Fig. 7E).

\section{DISCUSIÓN}

Los resultados indicaron que la proporción de sexos y la tasa de crecimiento poblacional (r) de jaiba café varían a lo largo de la costa de Sonora y que en el período reproductivo la
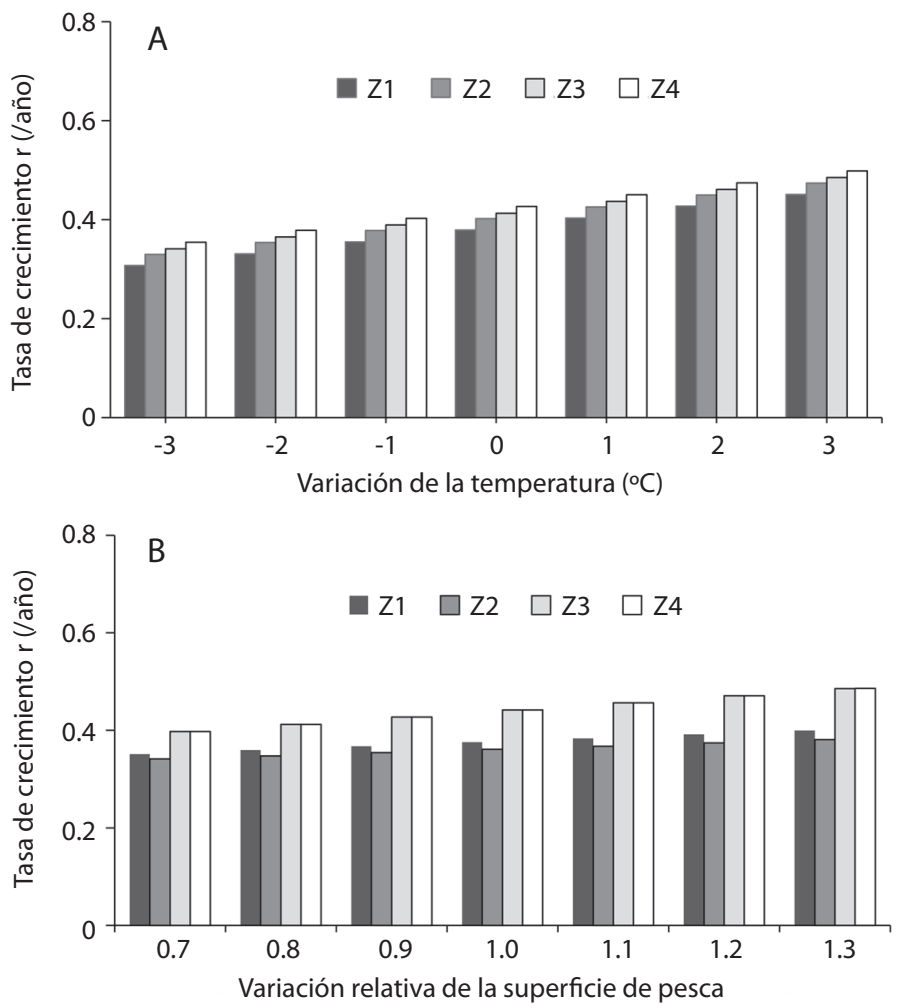

Fig. 6. Sensibilidad de la tasa de crecimiento poblacional de C. bellicosus por zona al variar: $\mathbf{A} . \pm 3^{\circ} \mathrm{C}$ la temperatura superficial del mar; y B. $\pm 30 \%$ la superficie de pesca. Resultados usando modelos de regresión lineal simple.

Fig. 6. Sensitivity of $C$. bellicosus population growth rate per zone varying: A. $\pm 30 \%$ sea surface temperature; and B. \pm 30 $\%$ the fishing surface. Results using simple linear regression models. 
CUADRO 4

Resultados de los modelos de regresión lineal simple (MRLS) y múltiple (MRLM) por zona

TABLE 4

Results for simple (MRLS) and multiple linear regression (MRLM) models by zone

\begin{tabular}{|c|c|c|c|c|c|c|c|}
\hline Zona & MRLS & $\mathrm{r}_{\mathrm{est}}$ & AIC & MRLM & $\mathrm{r}_{\mathrm{est}}$ & AIC & $\mathrm{w}_{\mathrm{i}}$ \\
\hline 1 & $\mathbf{a}$ & 0.40 & 18.777 & e & 0.42 & 26.802 & 12.4999 \\
\hline 2 & & 0.32 & & & 0.32 & & \\
\hline 3 & & 0.47 & & & 0.47 & & \\
\hline 4 & & 0.42 & & & 0.41 & & \\
\hline 1 & b & 0.38 & 16.481 & f & 0.4 & 26.772 & 12.4998 \\
\hline 2 & & 0.40 & & & 0.32 & & \\
\hline 3 & & 0.41 & & & 0.47 & & \\
\hline 4 & & 0.43 & & & 0.43 & & \\
\hline 1 & c & 0.36 & 17.435 & g & 0.4 & 26.77 & 12.4998 \\
\hline 2 & & 0.36 & & & 0.32 & & \\
\hline 3 & & 0.45 & & & 0.47 & & \\
\hline 4 & & 0.45 & & & 0.43 & & \\
\hline 1 & d & 0.38 & 17.300 & h & 0.3 & 37.21 & 12.5003 \\
\hline 2 & & 0.36 & & & 0.39 & & \\
\hline 3 & & 0.44 & & & 0.6 & & \\
\hline 4 & & 0.44 & & & 0.33 & & \\
\hline 1 & & & & i & 0.32 & 36.029 & 12.5003 \\
\hline 2 & & & & & 0.39 & & \\
\hline 3 & & & & & 0.6 & & \\
\hline 4 & & & & & 0.32 & & \\
\hline 1 & & & & $\mathbf{j}$ & 0.31 & 25.971 & 12.4996 \\
\hline 2 & & & & & 0.42 & & \\
\hline 3 & & & & & 0.46 & & \\
\hline 4 & & & & & 0.43 & & \\
\hline 1 & & & & $\mathbf{k}$ & 0.42 & 26.621 & 12.4998 \\
\hline 2 & & & & & 0.32 & & \\
\hline 3 & & & & & 0.47 & & \\
\hline 4 & & & & & 0.41 & & \\
\hline 1 & & & & 1 & 0.38 & 33.5 & 12.5003 \\
\hline 2 & & & & & 0.38 & & \\
\hline 3 & & & & & 0.58 & & \\
\hline 4 & & & & & 0.28 & & \\
\hline
\end{tabular}

$\mathrm{r}_{\text {est }}=$ tasa de crecimiento poblacional estimada por los modelos de regresión, AIC $=$ Criterio de Información de Akaike, $\mathrm{y}$ $\mathrm{w}_{\mathrm{i}}=$ peso relativo de los MRLM.

proporción de hembras tiende a incrementarse claramente en la parte central y aparentemente en la sur. Estudios anteriores reportaron segregación espacial por sexos (p. ej. Ramach, Darnell, Avissar, \& Rittschof, 2009); sin embargo, no se conocen trabajos publicados que documenten la variabilidad de $r$ en una misma área o la relación de $\mathrm{r}$ con la proporción de sexos o las condiciones del hábitat de las jaibas.

Pese a la escasez de datos, los resultados apuntan a conclusiones importantes sobre la biología y manejo pesquero de la jaiba café. La interpolación lineal y el uso de tendencias de valores contiguos pudieron alterar los valores 

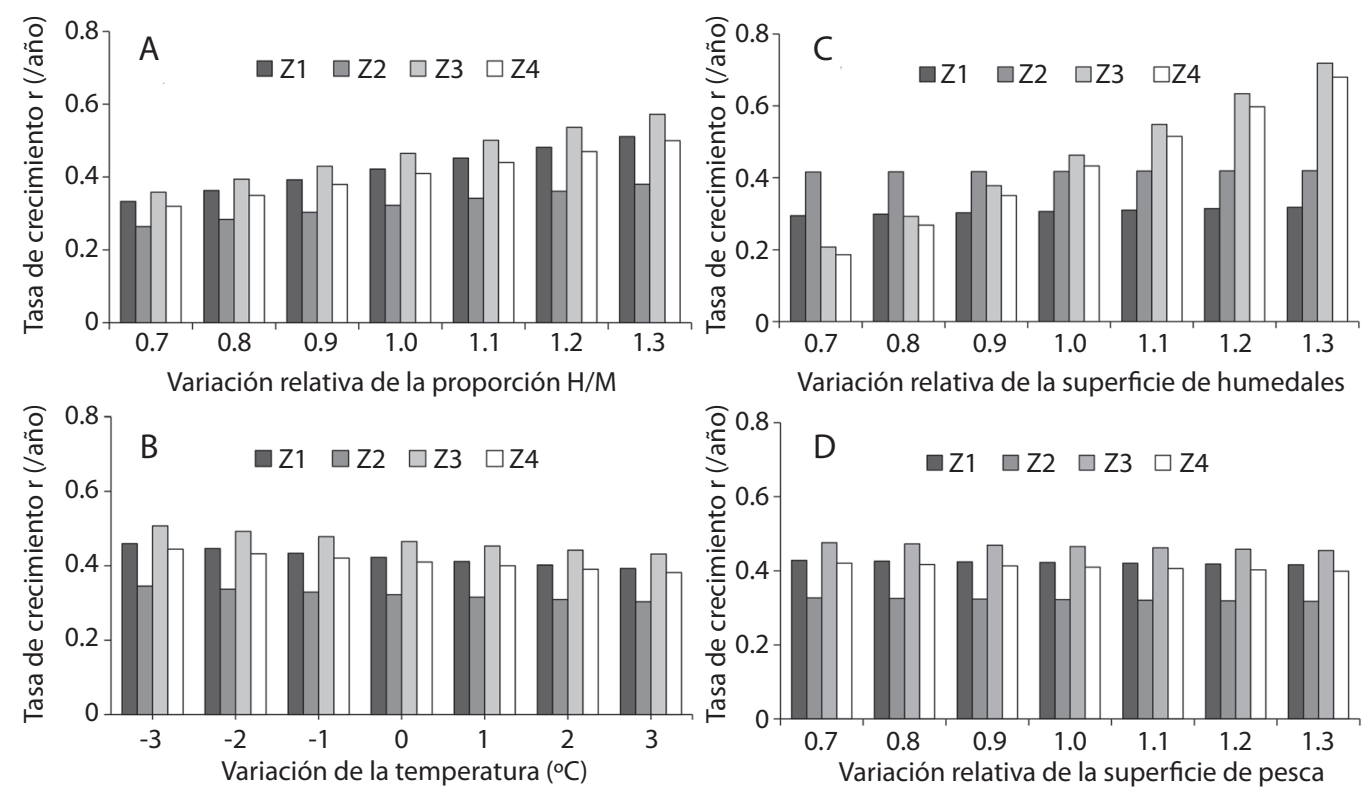

Fig. 7. Sensibilidad de la tasa de crecimiento poblacional de C. bellicosus al variar en cada zona: A. $\pm 30 \%$ la proporción H/M; B. $\pm 3{ }^{\circ} \mathrm{C}$ la temperatura del hábitat; C. $\pm 30 \%$ la superficie de humedales; y D. $\pm 30 \%$ la superficie de pesca. Resultados usando modelos de regresión lineal múltiple.

Fig. 7. Sensitivity of $C$. bellicosus intrinsic rate of population growth to variation in each zone: $\mathbf{A} . \pm 30 \%$ the ratio of $\mathrm{H} / \mathrm{M}$; B. $\pm 3{ }^{\circ} \mathrm{C}$ habitat temperature; C. $\pm 30 \%$ the surface of wetlands; and D. $\pm 30 \%$ the size of the fishing area. Results using multiple linear regression models.

reales de H/M. No obstante, en la zona 4 carente de datos de mayo a julio, H/M siguió la tendencia de la zona 3, para la cual hubo datos en el mismo período.

Se ignoran las razones por las cuales se observó mayor proporción de machos en las cuatro zonas. No se puede descartar un efecto del arte de pesca (Magana, Dobson, \& Mathooko, 2012) y posiblemente la proporción varíe incluyendo organismos de profundidades fuera del alcance de la pesquería. Los resultados coinciden con otros estudios de la misma especie dentro del Golfo de California recolectados con el mismo tipo de arte. En la laguna costera de Santa María La Reforma (Sinaloa) se encontró $71 \%$ de machos (Rodríguez, Castillo, Pérez, \& Aragón, 2012). En las bahías de Lobos y Las Guásimas (Sonora) la proporción de machos varió entre 55 y 57 \% (Nevárez et al., 2003; Reyes, López, Nevárez, \& Morales, 2007); en el Canal de Infiernillo-Bahía de Kino
(Sonora) la proporción de machos varió entre 64 y 85 \% (Bourillón, 2002; Torre, Bourillón, $\&$ Weaver, 2004).

La proporción de sexos 1:1 aumenta la probabilidad de éxito reproductivo (Fisher, 1930) y en crustáceos la proporción teórica es 1:1 (Warner, 1977), aunque puede variar. Las causas de la segregación por sexos incluyen: preferencia y disponibilidad de alimento; reducción del riesgo de depredación intraespecífica y del costo energético por apareamiento fuera de la época reproductiva; búsqueda de mejor hábitat; o diferentes tasas de mortalidad y crecimiento (Cobb \& Caddy, 1989; Cobb \& Wang, 1985; Heupel, Carlson, \& Simpfendorfer, 2007; Klimley, 1987; Sims, Nash, \& Marritt, 2001; Springer, 1967;).

En Callinectes la proporción de sexos varía temporalmente cuando las hembras ovígeras migran a la boca de las lagunas costeras para liberar los huevecillos, aunque después 
regresan al interior (Branco \& Masunari, 2000; Baptista et al., 2005). Por la muda terminal de las hembras de jaiba café (Rodríguez et al., 2012), la proporción de hembras disminuiría con la talla. La pesca sesgada hacia un sexo o tamaño, también podría hacer variar la proporción de sexos, ya sea en la población o en alguna zona.

Si la proporción dominante de machos de jaiba café observada en Sonora es algo natural, entonces para la población es más importante asegurar el suministro de esperma que maximizar la producción de huevos, y la biomasa debiera mantenerse estable o estar creciendo. Si la proporción natural es 1:1 o dominan las hembras, entonces es más importante la producción de huevos que el suministro de esperma, y la proporción observada reflejaría alteración de hábitat o sobrepesca de hembras; en ambos casos se esperaría que la biomasa fuera disminuyendo, como fue reportado (Cisneros et al., 2014).

Bajo el supuesto de que las condiciones del medio ambiente de cada zona influyen en $r$, los análisis de sensibilidad indicarían qué factores son preponderantes. Con información para solo cuatro zonas no fue posible desarrollar modelos estimativos sino indicativos. Los MRLM incluyeron variables sencillas y compuestas sin incurrir en colinealidad al no repetir variables independientes en el mismo MRLM. Los modelos b y k parecen contradecirse: usando el MRLS $b$ hubo una relación directa entre TSM y r, pero con el MRLM k no hubo respuesta a cambios en TSM. Esto denota efecto confusión de la TSM al asociarse con H/M en el MRLM $\mathrm{k}$. El otro efecto típico en regresión es la interacción, que ocurrió con SP en los MRLM: la variable $(\mathrm{H} / \mathrm{M}) / \mathrm{TSM}$, con un coeficiente (15.522) cinco órdenes de magnitud mayor que el de SP en ambos modelos, prácticamente nulificó el efecto de SP en el MRLM k. Derivado de los análisis, en teoría al tener mayor peso que las otras variables (individuales o combinadas) $\mathrm{H} / \mathrm{M}$ se relaciona directamente con r. Esto está en consonancia con lo observado para otros crustáceos: relación directa entre H/M y la actividad reproductiva (Serrano, 1991; Wilcox, 2007).

Los datos y los análisis indicaron que los humedales son importantes para los stocks de jaiba porque $\mathrm{H} / \mathrm{M}$ y $\mathrm{SH}$ se relacionan directamente con $\mathrm{r}$. El stock de la zona 3 tiene la mayor r, la mayor H/M y la mayor SH. Esto puede indicar que la producción de huevos aumenta con la abundancia de hembras (Lasker, 1985) y la abundancia de humedales favorece el reclutamiento (Carmona, 2009; De Lestang et al., 2010; Fischer \& Wolff, 2006). Dada la importancia de este posible hallazgo, en el futuro se deben incorporar mayor cantidad de datos para mejorar el análisis de sensibilidad.

Los resultados sugieren que el manejo de la pesquería de jaiba café en Sonora debe procurar proteger a las hembras. Esto se podría lograr estableciendo refugios pesqueros dentro $\mathrm{y}$ en las bocas de lagunas costeras durante el período de máxima actividad reproductiva (Kunsook, Gajaseni, \& Paphavasit, 2014). Una táctica similar se emplea exitosamente en la pesquería de langosta en Australia (Hall \& Chubb, 2001). Estudios posteriores debieran profundizar nuestros resultados y recomendar una proporción de sexos apropiada para la jaiba café de Sonora, porque la pesca excesiva de machos también podría afectar el reclutamiento (Botsford, 1995) y/o la variabilidad genética (Allendorf \& Hard, 2009).

\section{AGRADECIMIENTOS}

DRF agradece al CONACYT (beca 289169) y a COBI, A.C. por su apoyo económico. Gracias al personal del CRIP Guaymas por la recolecta de datos y a Edgar Alcántara por la obtención de TSM y el procesamiento de figuras. Enrique Morales, Antonio García, Evlin Ramírez y ocho revisores anónimos aportaron valiosas sugerencias para mejorar el presente artículo.

\section{RESUMEN}

Para la jaiba café (Callinectes bellicosus) capturada en Sonora, México dentro del Golfo de California desde 
1986, durante 20 años se ha observado una tendencia decreciente en la biomasa. Se estimaron las tasas de crecimiento poblacional (r) y proporción de sexos de $C$. bellicosus además de tres parámetros que describen su hábitat: temperatura del mar, extensión de humedales y tamaño del hábitat en cuatro zonas a lo largo de la costa de Sonora. La proporción de sexos se estimó de 25556 jaibas muestreadas de las capturas comerciales en los años 19982002 y 2012; la temperatura media en el período de desove (mayo-agosto) se generó a partir de sensores remotos en los mismos años; la cobertura de humedales se obtuvo de reportes publicados; y el tamaño del hábitat se estimó como la superficie de pesca. Para cada zona se estimó r mediante un método desarrollado para situaciones de escasa información utilizando las capturas comerciales (t) de 1986 a 2013. Con los datos de las cuatro zonas se desarrollaron modelos de regresión lineal simple y múltiple para evaluar las sensibilidades teóricas de $\mathrm{r}$ a variaciones en la proporción de sexos y en los parámetros ambientales. Los machos dominaron $(68.8 \%)$ sobre las hembras en el período de estudio en las cuatro zonas; un análisis de conglomerados identificó dos grupos de acuerdo a la proporción de sexos: un grupo norteño con las zonas 1 y 2 , y otro sureño con las zonas 3 y 4 . Los valores de $r$ fueron diferentes en las cuatro zonas $(\mathrm{P}<0.001)$ igual que la proporción de sexos $(\mathrm{P}=0.037)$; no se encontraron diferencias en la temperatura entre los años considerados en el estudio $(\mathrm{P}>0.995)$. Tanto los datos estimados como los análisis de sensibilidad sugieren que $r$ dependen directa y positivamente de la proporción de hembras de jaiba y el tamaño de los humedales. Por lo anterior planteamos la hipótesis de que la pesca excesiva de hembras es la causa del descenso de la biomasa de jaiba café en Sonora, y concluimos que es conveniente implementar refugios pesqueros dentro de humedales costeros para proteger las hembras en el período de desove.

Palabras clave: Callinectes bellicosus, proporción de sexos, tasa de crecimiento poblacional, Golfo de California.

\section{REFERENCIAS}

Allendorf, F. W., \& Hard, J. J. (2009). Human-induced evolution caused by unnatural selection through harvest of wild animals. Proceedings of the National Academy of Sciences, 106, 9987-9994.

Arreola, J. A. (1995). Diagnosis Ecológica de Bahía de Lobos, Sonora, México (Tesis de Maestría). Instituto Politécnico Nacional, La Paz, B.C.S., México.

Arreola, J. A., Hernández, L. G., Hernández, S., Flores, F., Lechuga, C., \& Ortega, A. (2003). Ecology of Callinectes arcuatus and C. bellicosus (Decapoda, Portunidae) in a coastal lagoon of northwest México. Crustaceana, 76, 651-664.

Ayala, A., Malpica, V. M., \& Gutiérrez, M. (1980). Geología Marina de la Región de Yavaros, Sonora, México.
Anales del Instituto de Ciencias del Mar y Limnología. Universidad Nacional Autónoma de México, 7 , 275-289.

Ayala, A., Gutiérrez, M., \& Malpica, V. M. (1990). Morfosedimentología de la laguna Agiabampo, SonoraSinaloa, México. Anales del Instituto de Ciencias del Mar y Limnología. Universidad Nacional Autónoma de México, 17, 257-270.

Baptista, C., Pinheiro, M. A., Blankensteyn, A., \& Borzone, C. A. (2005). Biologia populacional e reprodutiva de Callinectes danae Smith (Crustacea, Portunidae), no Belneário Shangri-lá, Pontal de Paraná, Paraná, Brasil. Revista Brasileira de Zoologia, 22, 446-453.

Beman, J. M., \& Christopher, A. (2006). Diversity of ammonia-oxidizing archaea and bacteria in the sediments of a hypernutrified subtropical estuary: Bahía del Tóbari, México. Applied \& Environmental Microbiology, 72, 7767-7777.

Botsford, L. W. (1995). Population dynamics of spatially distributed, meroplanktonic, exploited marine invertebrates. International Council for the Exploration of the Sea Marine Science Symposium, 199, 118-128.

Branco, J. O., \& Masunari, S. (2000). Reproductive ecology of the blue crab, Callinectes danae Smith, 1869 in the Conceição lagoon system, Santa Catarina Isle, Brazil. Revista Brasileira de Biologia, 60, 17-27.

Bourillón, L. (2002). Exclusive fishing zone as a strategy for managing fishery resources by the Seri Indians. Gulf of California, Mexico (Ph.D. Dissertation). University of Arizona, Tucson, Arizona.

Burnham, K. P., \& Anderson, D. R. (2002). Model selection and multimodel inference: A practical informationtheoretic approach. New York: Springer.

Canchola, A. C. (2009). Construcción de un canal navegable en el estero La Pinta Puerto Peñasco Sonora, México (Tesis Profesional). Instituto Politécnico Nacional, México, D.F.

Carver, A. M., Wolcott, T. G., Wolcott, D. L., \& Hines, A. H. (2005). Unnatural selection: Effects of a male-focused size-selective fishery on reproductive potential of a Blue crab population. Journal of Experimental Marine Biology and Ecology, 319, 29-41.

Carmona, C. A. (2009). Swimming crab community ecology in an estuarine complex in western Venezuela (Decapoda, Portunidae). Nauplius, 17, 19-27.

Castro, K. M., \& DeAlteris, J. T. (1988). Development of a swimming crab fishery in Ecuador. International Center for Marine Resource Development. The University of Rhode Island. November 1988. Fishery Development Support Service, USAID Working Paper, 23, 1-23. 
Cisneros, M. Á., Ramírez, E., García, J. A., Castañeda, V., Labastida, A., Gómez, C., \& Madrid, J. (2014). Pesca de jaiba en el litoral del Pacífico mexicano. México, D.F: Instituto Nacional de Pesca, SAGARPA.

Cobb, J. S., \& Caddy, J. F. (1989). The population biology of decapods. In J. F. Caddy (Ed.), Marine Invertebrate Fisheries: Their Assessment and Management (pp. 327-374). New York: John Wiley \& Sons.

Cobb, J. S., \& Wang, D. (1985). Fisheries biology of lobsters and crayfishes. In D. E. Bliss, (Ed.), The Biology of Crustacea (pp. 167-247). Orlando, Florida: Academic Press.

Cruz, V. A., \& Ortega, P. (1991). Modificación de la línea de costa como consecuencia del transporte litoral en el estero Tastiota, Sonora, México. Resúmenes del I Congreso Latinoamericano en Administración de la Zona Costera. México: Universidad Autónoma de Baja California. Ensenada, B.C.

De Lestang, S., Bellchambers, L. M., Caputi, N., Thomson, A. W., Pember, M. B., Johnston, D. J., \& Harris, D. C. (2010). Stock-recruitment-environment relationship in a Portunus pelagicus fishery in Western Australia. In G. H. Kruse, G. L. Eckert, R. J. Foy, R. N. Lipcius, B. Sainte-Marie, D. L. Stram, \& D. Woodby (Eds.), Biology and Management of Exploited Crab Populations under Climate Change (pp. 317-334). Alaska Sea Grant: University of Alaska, Fairbanks.

Diario Oficial de la Federación (DOF). (2006, Julio 26). NORMA Oficial Mexicana-039-PESC-2003. Pesca responsable de jaiba en aguas de jurisdicción federal del litoral del Océano Pacífico. Especificaciones para su aprovechamiento.

Diario Oficial de la Federación (DOF). (2012, Julio 11). Acuerdo por el que se modifica el Aviso por el que se da a conocer el establecimiento de épocas y zonas de veda para la pesca de diferentes especies de la fauna acuática en aguas de jurisdicción federal de los Estados Unidos Mexicanos, publicado el 16 de marzo de 1994 para establecer los periodos de veda de pulpo en el Sistema Arrecifal Veracruzano, jaiba en Sonora y Sinaloa, tiburones y rayas en el Océano Pacífico y tiburones en el Golfo de México.

Diario Oficial de la Federación (DOF). (2014, Julio 15). Acuerdo por el que se da a conocer el Plan de Manejo Pesquero de Jaiba (Callinectes spp.) de Sinaloa y Sonora, México.

Elmore, K., \& Richman, M. B. (2001). Euclidian distance as a similarity metric for principal component analysis. Monthly Weather Review, 129, 540-549.

Estrada, A. (1999). Aspectos poblacionales de la jaiba Callinectes arcuatus Ordway 1863, en la Laguna de Cuyutlán, Colima, México (Tesis de Maestría). Universidad de Colima, Colima, México.
Fisher, R. (1930). The genetic theory of natural selection. Oxford: Clarendon Press.

Fischer, S., \& Wolff, M. (2006). Fisheries assessment of Callinectes arcuatus (Brachyura, Portunidae) in the Gulf of Nicoya, Costa Rica. Fisheries Research, 77, 301-311.

Grabowski, R. C., Morilhas, S., \& Leão, A. (2014). Population structure, sex ratio and growth of the seabob shrimp Xiphopenaeus kroyeri (Decapoda, Penaeidae) from coastal waters of southern Brazil. ZooKeys, 457, 253-269.

Guzmán, M. C. (1993). Zonificación por medio de unidades ambientales de área contigua al estero del Soldado, Guaymas, Sonora, México (Tesis de maestría). Instituto Tecnológico y de Estudios Superiores de Monterrey Campus Guaymas, Guaymas, Sonora, México.

Hall, N. G., \& Chubb, C. (2001). The status of the western rock lobster, Panulirus cygnus, fishery and the effectiveness of management controls in increasing the egg production of the stock. Australian Journal of Marine and Freshwater Research, 52, 1657-1667.

Hammer, P., Harper, D. A. T., \& Ryan, P. D. (2001). PAST: Paleontological Statistics Software Package for Education and Data Analysis. Paleontologia Electronica, 4, 1-9.

Hendrickx, M. E. 1984. Studies of the coastal marine fauna of southern Sinaloa, Mexico. II. The decapod crustaceans of estero El Verde. Anales Centro de Ciencias del Mar y Limnología, Universidad Nacional Autónoma de México, 11, 23-48.

Heupel, M. R., Carlson, J. K., \& Simpfendorfer, C. A. (2007). Shark nursery areas: concepts, definition, characterization and assumptions. Marine Ecology Progress Series, 337, 287-297.

Hilborn, R., \& Mangel, M. (1997). The ecological detective. Confronting models with data. Monographs in Population Biology. Princeton, NJ: Princeton Academic Press.

Hilborn, R., \& Walters, C. J. (1992). Quantitative fisheries stock assessment. Choyce, dynamics \& uncertainty. New York: Chapman \& Hall.

Izar, J. M. (1998). Métodos numéricos para ingeniería. San Luis Potosí, México: Editorial Universitaria Potosina.

Klimley, A. P. (1987). The Determinants of sexual segregation in the scalloped hammerhead shark, Sphyrna lewini. Environmental Biology of Fishes, 18, 27-40.

Kunsook, C., Gajaseni, N., \& Paphavasit, N. (2014). A stock assessment of the blue swimming crab Portunus pelagicus (Linnaeus, 1758) for sustainable management in Kung Krabaen Bay, Gulf of Thailand. Tropical Life Sciences Research, 25, 41-59. 
Lasker, R. (1985). An egg production method for estimating spawning biomass of pelagic fish: Application to the Northern anchovy, Engraulis mordax. USA: National Oceanic and Atmospheric Administration. National Marine Fisheries Service.

Magana, A. M., Dobson, M., \& Mathooko, J. M. (2012) Modifying Surber sampling technique increases capture of freshwater crabs in African upland streams. Inland Waters, 2, 11-15.

Marinone, S. G., \& Lavín, M. F. (1997). Mareas y Corrientes residuales en el Golfo de California. In M. F. Lavín (Ed.), Contribuciones a la oceanografia física en México (pp. 113-139). México: Unión Geofísica Mexicana.

Martell, S., \& Froese, R. (2012). A simple method for estimating MSY from catch and resilience. Fish and Fisheries, 14, 504-514.

Nevárez, M. O., López, J., Cervantes, C., Miranda, E., Morales, R., \& Anguiano, M. L. (2003). Evaluación biológica y pesquera de las jaibas Callinectes bellicosus y Callinectes arcuatus (Brachyura: Decapoda: Portunidae) en las bahías de Guásimas y Lobos, Sonora, México. In M. E. Hendrickx (Ed.), Contributions to the study of East Pacific Crustaceans 2 (pp. 125-138). México: Instituto de Ciencias del Mar y Limnología, UNAM.

Orensanz, J. M., Armstrong, J., Armstrong, D., \& Hilborn, R. (1998). Crustacean resources are vulnerable to serial depletion-the multifaceted decline of crab and shrimp fisheries in the Greater Gulf of Alaska. Reviews in Fish Biology and Fisheries, 8, 117-176.

Ortega, P., \& Vázquez, D. A. (1989). Geoquímica de metales pesados y grado de piritización en tres lagunas costeras del Golfo de California, México. Resúmenes del III Congreso Venezolano sobre Ciencias del Mar. Cumaná, Venezuela: Instituto Oceanográfico de Venezuela, Universidad de Oriente.

Punt, A. E., \& Hilborn, R. (2001). BAYES-SA: Bayesian stock assessment methods in fisheries. User's manual. Computerized Information Series (Fisheries) No. 12. Rome: FAO.

Ramach, S., Darnell, M. Z., Avissar, N., \& Rittschof, D. (2009). Habitat use and population dynamics of blue crabs, Callinectes sapidus, in a high-salinity embayment. Journal of Shellfish Research, 28, 635-640.

Reyes, E., López, J., Nevárez, M. O., \& Morales, R. (2007). Dinámica poblacional de Callinectes bellicosus (Stimpson, 1859) y Callinectes arcuatus (Ordway, 1863) en Las Guásimas, Sonora, México, durante el periodo 2002 a 2004. In M. E. Hendrickx (Ed.) Contribuciones al Estudio de los Crustáceos del Pacífico Este (pp. 17-30). México: Instituto de Ciencias del Mar y Limnología, UNAM.
Reynolds, R. W., Rayner, N. A., Smith, T. M., Stokes, D. C., \& Wang, W. (2002). An improved in situ and satellite SST analysis for climate. Journal of Climate, $16,1609-1625$.

Rodríguez, G., Castillo, S. G., Pérez, R., \& Aragón, E. A (2012). Estimation of the individual growth parameters of the brown crab Callinectes bellicosus (Brachyura, Portunidae) using a multi-model approach. Crustaceana, 85, 55-69.

Sánchez, C. A., \& Gómez, J. (1992). Distribución y abundancia de los estadios planctónicos de la jaiba (Decapoda: Portunidae) en el complejo lagunar Bahía Magdalena, B.C.S., México. Revista de Investigación Científica de la Universidad Autónoma de Baja California Sur (Serie Ciencias del Mar), 3, 47-60.

Sato, T. (2012). Impacts of large male-selective harvesting on reproduction: illustration with large decapod crustacean resources. Aqua-BioScience Monographs, $5,67-102$

Schaefer, M. (1954). Some aspects of the dynamics of populations important to the management of the commercial marine fisheries. Bulletin of the InterAmerican Tropical Tuna Commission, 1, 27-56.

Serrano, A. V. (1991). Aspectos reproductivos de la langostilla Pleuroncodes planipes (Crustacea: Decapoda: Galatheidae) (Tesis de Maestría). Instituto Politécnico Nacional, La Paz, B.C.S., México.

Sims, D. W., Nash, J. P., \& Marritt, D. (2001). Movements and activity of male and female dogfish in a tidal sea lough: alternative behavioural strategies and apparent sexual segregation. Marine Biology, 139, 1165-1175.

Springer, S. (1967). Social organization of shark populations. In P. W. Gilbert, R. F. Metheson, \& D. P. Rall (Eds.). Sharks, Skates and Rays (pp. 149-174). Baltimore: Johns Hopkins Press.

Stearns, S. C. (1992). The evolution of life histories. New York: Oxford University Press.

Torre, J., Bourillón, L., \& Weaver, A. H. (2004). La Pesquería de la jaiba verde (Callinectes bellicosus) en la región de Bahía de Kino y Canal de Infiernillo entre 1998 y 2002. Informe Interno. Guaymas, Sonora, México: Comunidad y Biodiversidad, A. C. (COBI).

Warner, G. F. (1977). The biology of crabs. New York: Van Nostrand Reinhold.

Wilcox, W. M. (2007). Blue crab (Callinectes sapidus) ecology: review and discussion regarding Tisbury Great Pond (Martha's Vineyard Commission Report). Martha's Vineyard, Mass.

Zar, J. H. (1999). Bioestatistical Analysis. New Jersey: Prentice Hall. 
